\title{
Solving Anisotropic Transport Equation on Misaligned Grids
}

\author{
J. Chen ${ }^{1}$, S.C. Jardin ${ }^{1}$, and H.R. Strauss ${ }^{2}$ \\ 1 PPPL, P.O. Box 451, Princeton, NJ 08543, USA \\ ${ }^{2}$ Courant Institute, NYU, 251 Mercer Street, NY 10012, USA \\ jchen@pppl.gov
}

\begin{abstract}
Triangular 3rd order Lagrange elements have been implemented previously to study the numerical error associated with grid misalignment. It has previously been found that grid misalignment strongly affects numerical accuracy in the case of linear elements. The same conclusion was obtained by higher order finite difference. Here we observe that this is also true for higher order finite elements, up to 3rd order, when the solution has a steep gradient. Three types of meshes are considered. Type $t 1$ has one element edge fully aligned with the strong transport direction; type t2 doesn't have any alignment with that direction; type $\mathrm{t} 0$ is a combination of $\mathrm{t} 1$ and $\mathrm{t} 2$, i.e., partial alignment.
\end{abstract}

\section{Introduction}

Higher order Lagrange elements 1 have been used recently to study the numerical effect of grid misalignment 2] 3] on highly anisotropic transport problems. It has been found that the numerical solution is polluted by the grid misalignment when linear elements were used. Also in 4 it showed that grid alignment is critical to obtain accurate solution numerically even for higher order finite difference methods. In [1] we found that higher order elements can reduce the numerical artifacts caused by such misalignment significantly. Here we extend the study in [1] to another 2 types of meshes: $\mathrm{t} 1$ and $\mathrm{t} 2$. The mesh applied in [1] is a combination of $\mathrm{t} 1$ and $\mathrm{t} 2$, and we refer it as $\mathrm{t} 0$ for convenience.

The steady-state anisotropic heat conduction equation considered here is given in the orthogonal coordinates $(\xi, \eta)$ by:

$$
\frac{\partial}{\partial \xi} \kappa_{\xi} \frac{\partial T}{\partial \xi}+\frac{\partial}{\partial \eta} \kappa_{\eta} \frac{\partial T}{\partial \eta}=0
$$

Here $T$ is the temperature and $\kappa_{\xi}$ and $\kappa_{\eta}$ are the conductivities along the transport axes $\xi$ and $\eta$, respectively. Without loss of generality we assume $\kappa_{\eta} \equiv 1$ and $\kappa_{\xi} \gg 1$, so that the strong transport direction is aligned with $\xi$, and the anisotropy ratio is given by $\kappa_{\xi} / \kappa_{\eta} \equiv \kappa_{\xi}$.

The solution domain is given in the Cartesian coordinates $(x, y)$ by the rectangular ABCD: $\left[0, L_{x}\right] \times\left[0, L_{y}\right]$ with Dirichlet boundary conditions. As shown in Fig. 1. $[\mathrm{AB}]$ is aligned with the $\mathrm{x}$-coordinate and $[\mathrm{AD}]$ is aligned with the 


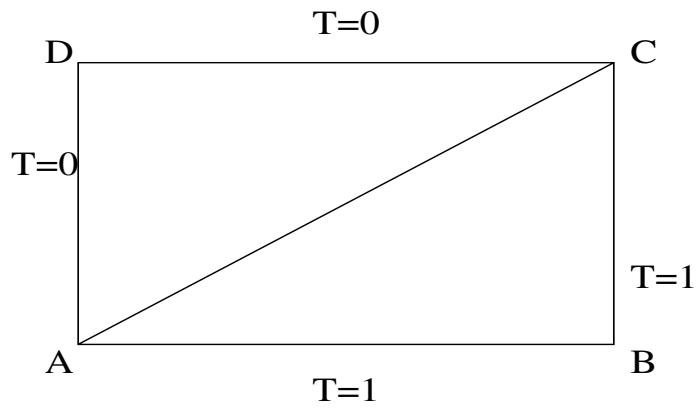

Fig. 1. Rect ABCD: $L_{x}=L_{y}=1 . \mathrm{T}=0$ on side $[\mathrm{AD}-\mathrm{DC}], \mathrm{T}=1$ on $[\mathrm{AB}-\mathrm{BC}]$. The strong anisotropic direction is aligned with the grid diagonal [AC]

$\mathrm{y}$-coordinate. The strong transport axis $\xi$ is aligned with the $[\mathrm{AC}]$ diagonal. The boundary conditions are: $\mathrm{T}=0$ on side $[\mathrm{AD}-\mathrm{DC}] ; \mathrm{T}=1$ on side $[\mathrm{AB}-\mathrm{BC}]$. For infinite anisotropy, the exact solution is: $T=0$ above the grid diagonal $[\mathrm{AC}]$, $T=1$ below the grid diagonal $[\mathrm{AC}]$, and the width of the transition zone is zero. For a finite anisotropy, the exact solution introduces an internal layer which has non-zero transition width.

\section{Mesh Setup}

The unstructured triangular mesh is formed by first dividing the rectangular domain ABCD into uniform rectangular cells: $\left[0, N_{x}-1\right] \times\left[0, N_{y}-1\right]$. Then each of the rectangle grids is subdivided into two triangles in the following 3 ways:

Mesh 11 in Fig. 2. The strong transport direction $\xi$ has full alignment with the element edge which is parallel to the diagonal $[\mathrm{AC}]$.

Mesh t2 in Fig. 2. The strong transport direction $\xi$ has no alignment with the element edge. The elements, aligned with $\xi$ int Mesh t1, are now aligned with the other diagonal [BD].

D

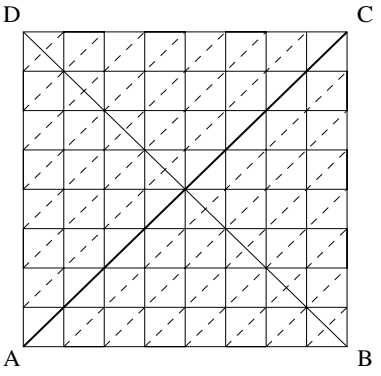

t1
C D

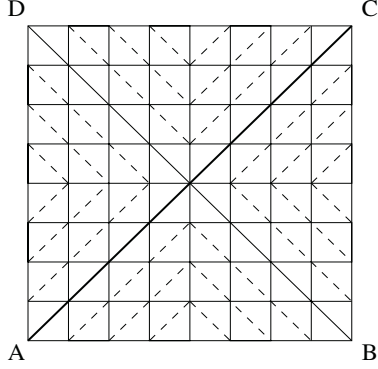

t0
$\mathrm{C} \quad \mathrm{D}$

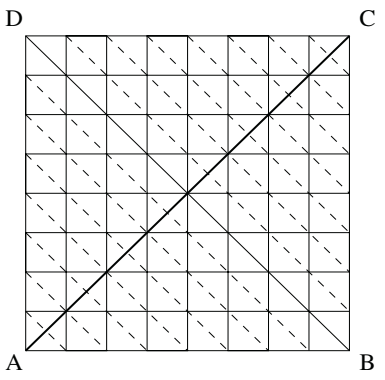

t2

Fig. 2. Meshes. $t 1: 100 \%$ alignment; $t 0: 50 \%$ alignment; $t 2: 0 \%$ alignment 
Mesh to in Fig. 2. This combines t1 and t2 such that the alignment is localized in the upper-right and lower-left blocks of the rectangular domain. In the upperleft and lower-right blocks, the misalignment is the same as in Mesh t2.

\section{$3 \quad$ Numerical Experiments and Discussions}

The numerical results for the convergence studies are presented in Table 1 and 2 for Mesh t1, t0 and t2, respectively in an ordering such that the alignment

Table 1. Profile width

\begin{tabular}{|c|c|c|c|c|c|c|c|c|c|c|c|c|}
\hline & \multicolumn{4}{|c|}{ t1 mesh ( $100 \%$ alignment) } & \multicolumn{4}{|c|}{ t0 mesh ( $50 \%$ alignment) } & \multicolumn{4}{|c|}{ t 2 mesh ( $0 \%$ alignment) } \\
\hline$N$ & $10^{2}$ & $10^{3}$ & $10^{4}$ & $10^{5}$ & $10^{2}$ & $10^{3}$ & $10^{4}$ & $10^{5}$ & $10^{2}$ & $10^{3}$ & $10^{4}$ & $10^{5}$ \\
\hline 39 & 0.1060 & 0.0353 & 0.0124 & 0.0094 & 0.1060 & 0.0354 & 0.0158 & 0.0138 & 0.1065 & 0.0441 & 0.0337 & 0.0328 \\
\hline 49 & 0.1061 & 0.0353 & 0.0121 & 0.0077 & 0.1059 & 0.0355 & 0.0141 & 0.0113 & 0.1063 & 0.0408 & 0.0289 & 0.0275 \\
\hline 59 & 0.1060 & 0.0350 & 0.0121 & 0.0066 & 0.1060 & 0.0351 & 0.0132 & 0.0096 & 0.1062 & 0.0386 & 0.0254 & 0.0240 \\
\hline 69 & 0.1060 & 0.0351 & 0.0117 & 0.0059 & 0.1060 & 0.0351 & 0.0125 & 0.0084 & 0.1062 & 0.0374 & 0.0226 & 0.0213 \\
\hline 79 & 0.1060 & 0.0350 & 0.0117 & 0.0054 & 0.1060 & 0.0350 & 0.0122 & 0.0076 & 0.1061 & 0.0365 & 0.0211 & 0.0193 \\
\hline 89 & & 0.0349 & 0.0115 & 0.0050 & & 0.0350 & 0.0119 & 0.0069 & 0.1061 & 0.0361 & 0.0196 & 0.0177 \\
\hline 99 & & 0.0349 & 0.0115 & 0.0047 & & 0.0350 & 0.0118 & 0.0063 & 0.1061 & 0.0357 & 0.0184 & 0.0163 \\
\hline 109 & & 0.0349 & 0.0115 & 0.0045 & & & 0.0117 & 0.0059 & & 0.0355 & 0.0174 & 0.0152 \\
\hline 119 & & & 0.0114 & 0.0043 & & & 0.0117 & 0.0055 & & 0.0353 & 0.0166 & 0.0143 \\
\hline 129 & & & 0.0114 & 0.0042 & & & 0.0116 & 0.0053 & & 0.0352 & 0.0159 & 0.0135 \\
\hline 139 & & & 0.0114 & 0.0040 & & & 0.0116 & 0.0051 & & 0.0351 & 0.0153 & 0.0127 \\
\hline 149 & & & 0.0114 & 0.0039 & & & 0.0115 & 0.0049 & & 0.0351 & 0.0147 & 0.0121 \\
\hline 159 & & & 0.0113 & 0.0039 & & & 0.0115 & 0.0048 & & 0.0350 & 0.0143 & 0.0116 \\
\hline 169 & & & 0.0113 & 0.0038 & & & 0.0114 & 0.0047 & & 0.0350 & 0.0139 & 0.0111 \\
\hline 179 & & & 0.0113 & 0.0037 & & & 0.0114 & 0.0046 & & 0.0350 & 0.0135 & 0.0106 \\
\hline 189 & & & & 0.0037 & & & 0.0114 & 0.0045 & & & 0.0133 & 0.0106 \\
\hline 199 & & & & 0.0038 & & & & 0.0044 & & & 0.0130 & 0.0099 \\
\hline 209 & & & & 0.0038 & & & & 0.0043 & & & 0.0127 & 0.0095 \\
\hline 219 & & & & 0.0038 & & & & 0.0042 & & & 0.0125 & 0.0092 \\
\hline 229 & & & & & & & & 0.0042 & & & & 0.0090 \\
\hline
\end{tabular}

Table 2. $L_{2}$ Norm

\begin{tabular}{|c|c|c|c|c|c|c|c|c|c|c|c|c|}
\hline & \multicolumn{4}{|c|}{ t1 mesh ( $100 \%$ alignment) } & \multicolumn{4}{|c|}{ t0 mesh (50\% alignment) } & \multicolumn{4}{|c|}{ t2 mesh (0\% alignment) } \\
\hline$N$ & $10^{5}$ & $10^{6}$ & $10^{7}$ & $10^{8}$ & $10^{5}$ & $10^{6}$ & $10^{7}$ & $10^{8}$ & $10^{5}$ & $10^{6}$ & $10^{7}$ & $10^{8}$ \\
\hline 39 & 0.9969 & 0.9972 & 0.9972 & 0.9972 & 0.9965 & 0.9967 & 0.9967 & 0.9967 & 0.9919 & 0.9919 & 0.9920 & 0.9920 \\
\hline 49 & 0.9974 & 0.9978 & 0.9978 & 0.9978 & 0.9971 & 0.9973 & 0.9974 & 0.9974 & 0.9933 & 0.9934 & 0.9934 & 0.9934 \\
\hline 59 & 0.9977 & 0.9981 & 0.9982 & 0.9982 & 0.9975 & 0.9978 & 0.9978 & 0.9978 & 0.9942 & 0.9943 & 0.9943 & 0.9943 \\
\hline 69 & 0.9979 & 0.9984 & 0.9985 & 0.9985 & 0.9977 & 0.9981 & 0.9981 & 0.9981 & 0.9949 & 0.9950 & 0.9950 & 0.9950 \\
\hline 79 & 980 & 0.9986 & 0.9987 & 0.9 & 979 & 0.9983 & 9983 & 83 & 9954 & 55 & 56 & 56 \\
\hline 89 & 9982 & 0.9987 & 0.9988 & 0.5 & 980 & 0.9985 & 0.9985 & 985 & 0.9958 & 0.9960 & 0.9960 & 0.9960 \\
\hline 99 & 9982 & 0.9988 & 0.9989 & 0.9989 & 0.9981 & 0.9986 & 0.9987 & 0.9986 & 0.9961 & 0.9963 & 0.9963 & 0.9963 \\
\hline 109 & .9983 & 0.9989 & 0.9990 & 0.9990 & 0.9982 & 0.9987 & 0.9988 & 0.9988 & 0.9964 & 0.9966 & 0.9966 & 0.9966 \\
\hline 119 & 0.9984 & 0.9990 & 0.9991 & 0.9991 & 0.9983 & 0.9988 & 0.9989 & 0.9989 & 0.9966 & 0.9968 & 0.9968 & 0.9968 \\
\hline 129 & 0.9984 & 0.9991 & 0.9992 & 0.9992 & 0.9983 & 0.9989 & 0.9989 & 0.9989 & 0.9968 & 0.9970 & 0.9970 & 0.9970 \\
\hline 139 & 0.9984 & 0.9991 & 0.9992 & 0.9992 & 0.9984 & 0.9989 & 0.9990 & 0.9990 & 0.9970 & 0.9972 & 0.9972 & 0.9972 \\
\hline 149 & 0.9985 & 0.9992 & 0.9993 & 0.9993 & 0.9984 & 0.9990 & 0.9991 & 0.9991 & 0.9971 & 0.9973 & 0.9974 & 0.9973 \\
\hline 159 & 0.9985 & 0.9992 & 0.9993 & 0.9993 & 0.9985 & 0.9990 & 0.9991 & 991 & 0.9972 & 0.9975 & 0.9975 & 0.9975 \\
\hline 169 & 0.9985 & 0.9992 & 0.9994 & 0.9994 & 0.9985 & 0.9991 & 0.9992 & 0.9992 & 0.9973 & 0.9976 & 0.9976 & 0.9976 \\
\hline 179 & 0.9985 & 0.9993 & 0.9994 & 0.9994 & 0.9985 & 0.9991 & 0.9992 & 0.9992 & 0.9974 & 0.9977 & 0.9977 & 0.9977 \\
\hline 189 & 0.9985 & 0.9993 & 0.9994 & 0.9994 & 0.9985 & 0.9992 & 0.9993 & 0.9993 & 0.9975 & 0.9978 & 0.9978 & 0.9978 \\
\hline 199 & 0.9986 & 0.9992 & 0.9995 & 0.9994 & 0.9985 & 0.9992 & 0.9993 & 0.9993 & 0.9976 & 0.9979 & 0.9979 & 0.9979 \\
\hline 209 & 0.9986 & 0.9993 & 0.9995 & 0.9995 & 0.9986 & 0.9992 & 0.9993 & 0.9993 & 0.9977 & 0.9980 & 0.9980 & 0.9980 \\
\hline 219 & 0.9986 & 0.9993 & 0.9995 & 0.9995 & 0.9986 & 0.9992 & 0.9994 & 0.9993 & 0.9977 & 0.9980 & 0.9981 & 0.9981 \\
\hline 229 & & 0.9994 & 0.9995 & 0.9995 & 0.9986 & 0.9993 & 0.9994 & 0.9994 & 0.9978 & 0.9981 & 0.9981 & 0.9981 \\
\hline 239 & & 0.9994 & 0.9996 & 0.9995 & & 0.9993 & 0.9994 & 0.9994 & 0.9978 & 0.9982 & 0.9982 & 0.9982 \\
\hline
\end{tabular}


is decreasing. The profile width, defined in [4], is used to detect the numerical convergence when $\kappa_{\xi}<=10^{5}$. If the width remains the same 3 times in a row when the grid resolution is increasing, we consider the solution converged. Only 3rd order elements are applied here based on the results from [1],

When the anisotropy $\kappa_{\xi}$ is small, $\leq 10^{3}$, as shown in columns 1 and 2 for each type of mesh in Table 1, the misalignment has a negligible effect. At $\kappa_{\xi}=10^{2}$, convergence takes place at grid resolution 79 for t1 and t0 meshes, and 99 for t 2 mesh. The solution on the t 2 mesh is within $0.1 \%$ of the one on the t 1 mesh. At $\kappa_{\xi}=10^{3}$, both solutions on the $\mathrm{t} 0$ and $\mathrm{t} 2$ meshes have deviated from the one on the $\mathrm{t} 1$ mesh by about $0.3 \%$, but convergence takes place later on the $\mathrm{t} 2$ mesh than on the t0 mesh.

When the anisotropy increases, the transition layer becomes thinner and the misalignment starts to come in and play a role.

At $\kappa_{\xi} \approx 10^{3}-10^{5}$, the misalignment has a medium effect. As seen from columns 3 and 4 in table 1 for the different types of mesh, the convergence takes place at a much earlier stage on the 11 mesh with full alignment. The convergence comes later on the partially aligned mesh t0, and it is seriously delayed on mesh t2 with no alignment. At $\kappa_{\xi}=10^{4}$, solutions on the t0 mesh has a deviation from the one on the $t 1$ mesh of about $0.8 \%$, but about $11 \%$ from the solution on the t2 mesh with no alignment. At $\kappa_{\xi}=10^{5}$, solutions on the t1 mesh converges at grid resolution 199, but not yet for solutions on the t0 and t2 meshes.

When the anisotropy continues to increase, $\kappa_{\xi}>10^{5}$, numerically it becomes difficult to measure the profile width correctly since this layer becomes very thin. Therefore, the $L_{2}$ norm of the solution is used in Table 2. This norm should converges to 1 when $\kappa_{\xi} \rightarrow \infty$. The data in Table 2 indicate that the alignment has a strong effect. The $L_{2}$ norm approaches 1 faster on the mesh, than the t0 mesh. The $L_{2}$ norm on the t2 mesh is the slowest. Therefore, we conclude that in order to achieve good precision for problems with high anisotropy, grid alignment is an important factor to be considered even for the 3rd order elements.

Finally, it is observed that $3 \mathrm{rd}$ order elements only works for $\kappa_{\xi}<=10^{7}$ since the $L_{2}$ norm remains the same at $\kappa_{\xi}=10^{7}$ and $\kappa_{\xi}=10^{8}$. For problem with $\kappa_{\xi}>10^{7}$, higher order elements than 3 rd order should be introduced. We will address this in the future.

\section{References}

1. J. Chen et al, Higher Order Lagrange Elements in M3D, DOE Technical Report, 2005. http://www.osti.gov/servlets/purl/836490-CNwldj/native/.

2. R. Vesey and D. Steiner, A Two-Dimensional Finite Element Model of the Edge Plasma, J. Comp. Phys. 116 300-313 (1994).

3. R. Zanino, Advanced Finite Element Modeling of the Tokamak Plasmas Edge, J. Comp. Phys. 138 881-906 (1997).

4. M. V. Umansky, M. S. Day, and T. D. Rognlien, On Numerical Solution of Strongly Anisotropic Diffusion Equation on Misaligned Grids. Submitted to Numerical Heat Transfer. 